\title{
Vicios, sesgos y conjuros arbitrales
}

\author{
Xavier Andrade Cadena* \\ Carolina Arroyo Aguirre** \\ Estefanía Fierro Valle*** \\ Recibido/Received: 29/07/2017 \\ Aceptado/Accepted: 31/07/2017
}

SUMARIO: 1. Introducción. 2. Introducción vs. cognición: dos sistemas arbitraje y la acción extraordinaria de protección. 3. Sesgos recurrentes 3.1 Sesgo de retrospectiva. 3.2 Anclaje. 3.3 Sesgos egocéntricos. 3.4 Aversión a los extremos. 4. ¿Cómo mejorar las decisiones arbitrales? 4.1 Controlar el tiempo. 4.2 Razones con el Sistema. 4.3 Redactar el laudo minuciosamente. 5. Conclusiones.

PALABRAS CLAVE: vicios, sesgos, Sistema 1, Sistema 2, psicología.

KEYWORDS: defect, biases, System 1, System 2, psychology.

RESUMEN: Debido a la compleja interacción entre los componentes intuitivo y analítico del pensamiento humano, los procesos cognitivos que llevan a cabo los juzgadores pueden estar sometidos a distintos sesgos cognitivos. Este artículo

* Socio de la firma Andrade Veloz, Profesor de la Universidad San Francisco de Quito. LL.B. y J.D. por la Pontificia Universidad Católica del Ecuador, LL.M. por McGill University, M.A. in Economics for Competition Law por King's College London.

** Asociada Senior de la firma Andrade Veloz. LL.B. y B.A. en Administración de Empresas por la Universidad San Francisco de Quito, MLB. por Bucerius Law School, WHU Otto Beisheim School of Management.

*** Asociada de la firma Andrade Veloz, Profesora adjunta de la Universidad San Francisco de Quito. LL.B. por la Universidad San Francisco de Quito. 
busca aplicar la nueva era de estudios psicológicos y neurocientíficos a la resolución de conflictos, a través de una guía con las técnicas que se podrían emplear para neutralizar los sesgos y mejorar las decisiones arbitrales.

ABSTRACT: Due to the complex interaction between the intuitive and analytical components of human thinking, the cognitive processes performed by the judges and arbitrators may be subject to different cognitive biases. This article seeks to apply the new era of psychological and neuroscientific studies to conflict resolution, through a guide with techniques that could be used to neutralize biases and improve arbitral decisions.

\section{INTRODUCCIÓN}

Varias escuelas de pensamiento han desarrollado modelos sobre el proceso cognitivo que realiza un juzgador. En un extremo, encontramos a los formalistas, quienes consideran que los jueces y árbitros deben aplicar el derecho a los hechos, de manera lógica, mecánica y deliberada. Para ellos, el sistema de administración de justicia es una especie de "máquina silogística gigante" operada por un mecánico altamente capacitado: el juzgador ${ }^{1}$.

En el otro extremo, se encuentran los realistas, para quienes el juicio sigue un ciclo intuitivo hasta llegar a las conclusiones, las cuales son racionalizadas solo en un momento posterior. Para esta escuela, los juzgadores deciden sintiendo, no juzgando, sobre la base de sus corazonadas, no de su raciocinio. La deliberación se utiliza solo para justificar la intuición ${ }^{2}$.

1. B. Neuborne, "Of Sausage Factories and Syllogism Machines: Formalism, Realism, and Exclusionary Selection Techniques, N.Y.U. Law Review, No. 67, 1992, p. 421. Citado por B. LeITER, "Positivism, Formalism, Realism”, Columbia Law Review, No. 99, 1999, p. 1145, «https://goo.gl/Dhe51i».

2. Véase, por ejemplo, J. C. Hutcheson JR., "Judgment Intuitive: The Function of the 'Hunch' in Judicial Decision”, Cornell Law Review, No. 14, 1929, «https://goo.gl/Az7Pkb». 
Sin embargo, la nueva ola de estudios psicológicos demostraría, al menos inicialmente ${ }^{3}$, que el proceso cognitivo de los juzgadores no es formalista ni realista, sino una amalgama de los dos modelos. En este contexto, GUTHRIE, RACHLINSKI y WISTRICH proponen un sistema "menos idealista que el formalista y menos cínico que el realista", bautizado por estos autores como el "formalismo realista" ${ }^{4}$.

El formalismo realista encontraría sustento en estudios recientes, como los publicados por el premio Nobel DANIEL KAHNE$\mathrm{MAN}^{5}$, quien postula que la mente humana tiene un sistema intuitivo y otro analítico, como se explicará más adelante.

El problema que aborda este ensayo tiene que ver con los vicios y sesgos que podrían aquejar al sistema intuitivo de un juzgador. Para analizar esta problemática, iniciaremos explicando brevemente cómo funcionan los sistemas intuitivo y analítico del juzgador (Sección 2). Luego, expondremos los sesgos más comunes que se han observado en la resolución de disputas (Sección 3) y los conjuros que se proponen para combatirlos (Sección 4). Finalmente, arribaremos a ciertas conclusiones (Sección 5).

\section{INTUICIÓN VS. COGNICIÓN: DOS SISTEMAS}

La idea de que la mente humana tiene un componente intuitivo y otro analítico ha sido desarrollada consistentemente a través de los tiempos. Platón, por ejemplo, para explicar las decisiones de las personas, proyectaba la imagen de dos caballos: uno bueno, gobernado por la razón, y otro malo y descarriado, conducido por la violencia ${ }^{6}$. DESCARTES también propuso que la intuición y la deducción eran la vía para arribar al conocimiento ${ }^{7}$.

3. Existe el consenso entre los académicos de que el estado de la ciencia en esta materia es inicial.

4. C. Guthrie, J. Rachlinski y A. Wistrich, "Blinking on the Bench: How Judges Decide Cases", Cornell Law Faculty Publications, Paper 917, 2007, p. 3, «https://goo.gl/GHTf7Q».

5. D. Kahneman, Pensar rápido, pensar despacio, Debolsillo, 2013.

6. Platón, Phaedrus, Christopher Rowe, 2005, pp. 34-36. Citado por E. Sussman, “Arbitrator Decision Making: Unconscious Psychological Influences and What You Can Do About Them”, American Review of International Arbitration, Vol. 3, 2013, pp. 488-489, «https://goo.gl/1Ke5ga».

7. Descartes. Citado por E. Sussman, N. 6. 
En el mismo sentido, PASCAL hablaba de dos tipos de mentes: la intuitiva y la geométrica, siendo la segunda la encargada de verificar los presupuestos de la primera ${ }^{8}$.

Más recientemente, DANIEL KAHNEMAN, a fin de explicar la complejidad del pensamiento humano, presenta a la mente como si estuviere dividida en dos sistemas: el 1 y el 2. El Sistema 1 opera de manera rápida, automática, intuitiva. El Sistema 2 opera de manera lenta, compleja, analítica.

Así, el Sistema 1 se activa, por ejemplo, para responder a preguntas como $i 2+2$ ? o ¿cuál es la capital de Francia? Sirve también para interpretar las señales de nuestros sentidos, evitar peligros, masticar y realizar otras tareas que se desarrollan de manera automática, aunque podamos controlarlas.

El Sistema 2, por su parte, realiza actividades más complejas y que requieren atención. Por ejemplo, se activa al escuchar la conversación de la mesa de al lado, realizar un silogismo jurídico o calcular $357 \times 15$.

Distinguir estos dos sistemas es relevante porque, pese a que ambos se encuentran activos, el Sistema 1 envía permanentemente sugerencias al Sistema 2 a modo de intuiciones, intenciones y sensaciones. En vista de que el Sistema 1 suele tener "buen juicio" -y por eso confiamos en nuestra intuición y "sentido común"- el Sistema 2 acoge la mayoría de sus sugerencias. Es el Sistema 2 el que en todo caso tiene la última palabra.

Pese a la confiabilidad del Sistema 1, su rapidez y superficialidad podrían dar lugar a errores sistemáticos que nos conducirían a decisiones equivocadas. Esta reflexión es útil, no sólo para comprender las siguientes secciones de este artículo sino para aplicarla a nuestra práctica profesional, sea que actuemos como juzgadores o litigantes.

8. Pascal. Citado por C. Guthrie, J. Rachlinski y A. Wistrich, N. 4, p. 6. 
Por ejemplo, por un lado, un árbitro estará mejor preparado para dictar un laudo si conoce que su intuición podría llevarle a tomar decisiones equivocadas. Este ensayo propone algunas técnicas para mitigar estos riesgos.

Por otro lado, un litigante podría presentar de manera más convincente su caso si entiende cómo funciona la mente del juzgador. Por ejemplo, existen casos en que los litigantes podrían preferir que el tribunal mantenga su pensamiento en el Sistema 1 o 2. Si un abogado presenta un caso complejo ante un tribunal arbitral, cuya primera apariencia no le beneficia (por prejuicios, sesgos o facilidad del caso para la contraparte, etc.), podría intentar llevar al tribunal a su Sistema 2, esto es, a un proceso más analítico. En contraste, si un proceso analítico develaría las falencias de su caso, el abogado podría intentar que sus argumentos apunten a influir en el Sistema 1 del tribunal (mediante argumentos emocionales, por ejemplo).

Es necesario anticipar que estas técnicas están lejos de ser científicamente comprobadas, pues el proceso cognitivo de un tribunal arbitral es supremamente complejo; sin embargo, la simple idea de que podamos mejorar nuestra práctica conociendo cómo funciona la mente de los humanos, invita a pensar en un universo de técnicas de litigio (que, en ocasiones, incluso parecerían invadir la intimidad del pensamiento del juzgador).

Aplicar la nueva era de estudios psicológicos y neuro-científicos a la resolución de conflictos es un ejercicio fascinante. Hace casi un siglo el juez CARDOzO anticipaba que:

Mucho más allá de la conciencia, existen otras fuerzas, gustos y aversiones, predilecciones y prejuicios, una serie de instintos y emociones, hábitos y convicciones, que hacen al hombre, sea juez o litigante'.

9. Traducción libre. B. CARdozo, The Nature of the Judicial Process, Yale University Press, 1921. Citado por E. Sussman, N. 6, p. 490. 
En el mismo sentido, el juez de la Corte Suprema estado-unidense, ANTONIN SCALIA, señaló que:

Mientras las computadoras funcionan solo de manera lógica, los seres humanos no funcionan así. Toda una serie de factores extraños -emociones, sesgos, preferencias- pueden intervenir [en el proceso cognitivo], y acerca de la mayoría de ellos no podemos hacer absolutamente nada (salvo jugar con ellos, si se los llega a conocer) ${ }^{10}$.

\section{Sesgos ReCURRENTES}

Los sesgos cognitivos son procedimientos de simplificación que usa nuestro cerebro para procesar la complejidad de la información. Estos sesgos se producen de manera inevitable, por el simple hecho de que usamos nuestro cerebro para procesar la información que recibimos del mundo exterior. Como explican TVERSKY y KAHNEMAN, estas operaciones cognitivas tienden a "reducir las tareas complejas de asignar probabilidad y predecir valores a operaciones de juicio más simples"11.

En atención a la naturaleza subjetiva (y a veces involuntaria) del proceso cognitivo de los juzgadores (sean estos jueces o árbitros), en esta sección identificaremos los sesgos que, según estudios psicológicos, afectarían recurrentemente a la resolución de disputas.

\subsection{Sesgo de retrospectiva}

Una vez que ocurre un fenómeno o evento, es difícil evaluar objetivamente si este era previsible o no. Los estudios demuestran que la gente suele exagerar la previsibilidad de un evento que $e x$ ante podría no ser previsible. Como explican GUTHRIE, RACHLINSKI y WiSTRICH, "la gente sobrestima su propia habilidad para prede-

10. Traducción libre. A. Scalia y B. Garner, Making Your Case: The Art of Persuading Judges, Thomson West, 2008, Introducción, p. xxiii.

11. A. TVersky y D. Kahneman, "Judgment under Uncertainty: Heuristics and Biases", Science, New Series, Vol. 185, No. 4157, 1974, pp. 1124-1131, «https://goo.gl/faJFnr». 
cir el pasado y creen que otras personas deberían haber sido capaces de predecir eventos de mejor manera que la [que realmente] era posible"12.

El ejemplo clásico de este sesgo fue presentado por KAMIN y RACHLINSKI ${ }^{13}$. El experimento versa sobre una ciudad cuyo puente levadizo opera únicamente de primavera a otoño. Se preguntó a dos grupos de personas si el riesgo de inundación en esta ciudad era tan alto como para contratar a un operador de puentes durante el invierno. A uno de los grupos se les dijo que si la ciudad hubiese contratado un operador de puentes durante el invierno se habría evitado que el fondo del río se sedimente y cause una inundación. El otro grupo no conocía esta información. En el grupo que conocía que existió una inundación (i.e. tenían información retrospectiva), 57\% de las personas consideraron que la ciudad fue negligente al no contratar a un operador de puentes, mientras que únicamente el $24 \%$ de las personas del otro grupo consideraron que tal contratación era necesaria.

Este sesgo es relevante para la resolución de conflictos, pues los árbitros a menudo deben evaluar un fenómeno de manera retrospectiva, es decir, una vez que el hecho ha sucedido. En estos casos, los árbitros suelen estar compelidos a valorar, por ejemplo, si el efecto fue generado por una determinada causa (i.e. nexo causal); si las partes fueron lo suficientemente diligentes para evitar el evento; si el evento era razonablemente previsible ${ }^{14}$; si la experiencia de las partes era relevante para tal previsibilidad; si las partes revelaron información suficiente o si la analizaron con la debida diligencia; entre otras cuestiones usuales sobre la causalidad de un evento y sus consecuencias económicas y jurídicas.

Dado que estos juicios de valor suelen implicar la cuantificación de una indemnización, la responsabilidad del árbitro de evi-

12. Traducción libre. C. Guthrie, J. Rachlinski y A. Wistrich, "Inside the Judicial Mind", Cornell Law Faculty Publications, Paper 814, 2001, p. 799, «https://goo.gl/P75Df8».

13. K. Kamin y J. Rachlinski, "Ex Post $\neq$ Ex Ante: Determining Liability in Hindsigh”, Cornell Law Faculty Publications, Paper 646, 1995, «https://goo.gl/9oTSce».

14. C. Drahozal, "A Behavioral Analysis of Private Judging", Law and Contemporary Problems, Vol. 67(1) 105-132, 2004, p. 110, «https://goo.gl/NZ4Ufn». 
tar el sesgo de retrospectiva es latente. Como señalan KAMIN y RACHLINSKI:

En vista de que los litigios de responsabilidad civil implican necesariamente valoraciones ex post, toda ausencia de diligencia de una persona podría dar lugar a un caso de responsabilidad [civil], incluso en casos en que tal diligencia no hubiese sido justificable ex ante ${ }^{15}$.

Adicionalmente, el sesgo de retrospectiva podría verse alimentado con la experiencia propia de cada árbitro. Una vez que un abogado conoce un caso, sea en calidad de litigante o de árbitro, su mente difícilmente puede olvidarlo y, como resultado, su sesgo de retrospectiva podría influir en la valoración de la predictibilidad de un evento. Ahora, este sesgo no es necesariamente nocivo mientras se lo tenga presente y no se permita que se apodere de la decisión del juzgador. No se puede pedir a un árbitro que elimine de su bagaje psicológico toda su experiencia -que es una de las razones por las que podría haber sido seleccionado por las partes-. Lo que se propone es que no se deje engañar por su mente.

\subsection{Anclaje}

Cuando las personas calculamos un monto desconocido, tenemos la tendencia a definirlo sobre la base del primer valor que se nos presenta. Este valor inicial o "ancla"16 es comúnmente el punto de partida de los cálculos subsecuentes. Para definir el monto final, solemos hacer ajustes a esa ancla con base en ciertos parámetros que a veces no son reales o suficientes. Como explican los psicólogos STRACK y MussweILER, los números que se toman en consideración en una etapa inicial tienen mayor impacto en el cálculo que cualquier otra información numérica que se obtenga posteriormente ${ }^{17}$.

15. Traducción libre. K. KAmin y J. RachlinsKi, N. 13.

16. Sobre el fenómeno del anclaje, véase generalmente A. TVERSKY y D. KAHNEMAN, N. 11.

17. F. Strack y T. Mussweiler, "Explaining the Enigmatic Anchoring Effect: Mechanisms of Selective Accessibility", Journal of Personality and Social Psychology, Vol. 73(3), 1997, pp. 437-446, «https://goo.gl/KdJnBo». 
Por ejemplo, si entramos a una tienda para adquirir un televisor, y el vendedor nos presenta dos opciones: una de US\$ 200 y otra de US\$ 300, es posible que nuestra ancla se fije en el promedio de los dos valores, es decir en US\$250. Imaginemos ahora que, antes de tomar la decisión de compra, el vendedor de repente nos propone una tercera opción de US\$ 400. Es probable que nuestra ancla cambie al promedio de los tres precios: US\$ 300. Esto hará que el televisor de US\$ 300 ya no nos parezca tan caro como antes ${ }^{18}$.

Otro ejemplo presentado por DANIEL KAHNEMAN explica que si "a alguien se le pregunta si Gandhi tenía más de ciento cuarenta años cuando murió, terminará haciendo una estimación más alta de la edad a [la] que murió, de la que habría hecho si la pregunta ancla hubiera hablado de 35 años"19. Para KAHNEMAN, los resultados del efecto ancla son de los "más fiables y sólidos de la psicología experimental" 20 y están presentes en un sinnúmero de ocasiones cotidianas.

Este sesgo tiene especial relevancia para los casos en que se reclaman indemnizaciones por daños y perjuicios. De hecho, existen varios estudios que demuestran que las decisiones de los juzgadores se encuentran "ancladas" a los montos que las partes proponen. Por ello, una demanda que propone un monto de indemnización desmesuradamente alto o bajo podría anclar la mente del árbitro y causar que la indemnización que ordene sea demasiado alta o baja.

Por ejemplo, GUTHRIE, RACHLINSKI y WISTRICH demostraron fehacientemente el sesgo de anclaje encuestando a ciento sesenta y cinco experimentados jueces de los EE.UU. ${ }^{21}$. A un grupo de jueces se le preguntó sobre el monto de daños que concedería al demandante en un caso de negligencia en el que perdió una pierna, sin mencionar ningún parámetro de daños o cifras numé-

18. Ejemplo modificado al originalmente provisto por I. SiMONSON y A. TVERSKY. "Choice in Context: Tradeoff Contrast and Extremeness Aversion”, Journal of Marketing Research, 1992, «https://goo.gl/DnGRdN».

19. D. Kahneman, Pensar rápido, pensar despacio, N. 5, p. 162.

20. Ibídem.

21. C. Guthrie, J. Rachlinski y A. Wistrich, "Inside the Judicial Mind”, N. 12 (2001), pp. 791-792. 
ricas. Al segundo grupo, en contraste, se le informó que si el monto de la indemnización fuere menor a US\$75.000, el demandante no tendría acceso a la jurisdicción de las cortes federales (por tratarse de montos menores). Como resultado, los jueces del primer grupo, que no tenían un ancla numérica en mente, otorgaron en promedio una indemnización de US\$1'249.000, mientras que el promedio del segundo grupo fue de solo US\$ 882.000; es decir, casi la tercera parte del promedio del primer grupo. Este experimento demuestra que el hecho de que los jueces hayan tenido un monto en su mente afectó sustancialmente el valor de la indemnización otorgada.

En vista de que en la mayoría de arbitrajes existe una reclamación de daños y perjuicios (pues son raros los casos en los que únicamente se busca la declaración de un derecho), el sesgo de anclaje jugaría un rol relevante en la definición de los montos indemnizatorios. Dado que la determinación equívoca de indemnizaciones comprometería la calidad de la justicia, provocando resultados potencialmente injustos, es prudente sugerir que los árbitros tomen medidas para no ser presas de sus sesgos. Estas reflexiones también son útiles para los abogados litigantes, quienes deberían tener presente el efecto de anclaje que causa la determinación de cuantías en sus casos.

\subsection{SeSGOS EgOCÉNTRICOS}

Las personas no siempre somos objetivas respecto de nuestras habilidades y buen juicio. Tendemos a juzgarnos de manera egocéntrica. Por ejemplo, la mayoría de personas considerará que es un buen piloto (en comparación con el promedio de pilotos); las parejas recién casadas afirmarán que la probabilidad de que se divorcien es casi nula (a pesar de que la tasa de divorcios sea de aproximadamente $50 \%)^{22}$; y, la gran mayoría de profesores se considerarán mejores al profesor promedio ${ }^{23}$.

22. L. BAKER y R. EMERY, "When Every Relationship is Above Average: Perceptions and Expectations of Divorce at the Time of Marriage", Law \& Human Behavior, Vol. 17 (4), 1993. Citado por C. GuthriE, J. RACHLINSKI y A. Wistrich, N. 12 (2001), p. 812.

23. Ídem, p. 818. 
Los sesgos egocéntricos pueden afectar la práctica de los litigantes y árbitros. Por ejemplo, los litigantes tienden a creer que su caso es mejor de lo que realmente es, lo cual podría comprometer la posibilidad de alcanzar un acuerdo negociado. Si un litigante está convencido de la fortaleza de su caso, no encontrará suficientes incentivos para llegar a un acuerdo con la contraparte por una cifra menor a la demandada. Como resultado, si la predicción del abogado es irreal, el cliente se vería perjudicado.

Los juzgadores sufren del mismo sesgo. Por ejemplo, en una investigación realizada por GUTHRIE, RACHLINSKI y WISTRICH, se consultó a un grupo de ciento cincuenta y cinco jueces estadounidenses sobre la posibilidad de que sus fallos sean revertidos en una instancia de apelación. Más del 56\% de los jueces consideraron que la posibilidad de que sus fallos sean revertidos era mínima $^{24}$. La realidad, sin embargo, era distinta.

El problema con los sesgos egocéntricos es que los juzgadores podrían no estar conscientes de sus limitaciones y la posibilidad de cometer errores. En la práctica, estos sesgos podrían propiciar que los árbitros confíen demasiado en su propio juicio y no tomen en cuenta pruebas o argumentos relevantes de las partes. El exceso de confianza también podría impedir que un árbitro reconozca un error y revise su decisión, en detrimento de la justicia ${ }^{25}$.

Otro efecto relevante del sesgo egocéntrico es el juicio intuitivo que los árbitros podrían formarse luego de recibir los primeros memoriales de las partes o después de tener la primera audiencia, impidiendo que su mente analice objetivamente todas las pruebas y el resto de argumentaciones. Por ejemplo, un estudio realizado en arbitraje internacional demostró que al menos el $50 \%$ de los árbitros consultados aceptaron haberse creado un juicio inicial sobre el caso luego de leer los primeros memoriales y antes de tener la primera audiencia ${ }^{26}$. Esto podría

24. Ídem, pp. 811-816.

25. L. ReED, “Arbitral Decision-making: Art, Science or Sport?”, The Kaplan Lecture 2012, «https://goo.gl/fwijEF».

26. E. Sussman, “Arbitrator Decision Making: Unconscious Psychological Influences and What You Can Do About Them”, N. 6, p. 500. 
constituir una evidencia de que los árbitros confían (en exceso) en su Sistema 1.

Respecto a esta problemática, conviene citar las impresiones de los abogados y psicólogos WAITES y LAWRENCE acerca del proceso cognitivo de los árbitros:

Un árbitro típico concluye la fase inicial [del arbitraje] con una sola historia dominante en su mente [...]. Un porcentaje considerable de árbitros se habrán formado una clara inclinación al momento en que concluyan los alegatos iniciales (y antes de conocer las declaraciones de testigos o las pruebas). Esto significaría que para la mayoría de árbitros, el arbitraje es un proceso de filtración, a través de las pruebas, que tiene por objeto comprobar su propia hipótesis sobre el caso, con miras a confirmar o alterar su noción inicial sobre la realidad del caso...Los árbitros...harán lo posible por adaptar su percepción de los hechos a la historia que se han formado ${ }^{27}$.

Los riesgos que pueden generar los sesgos egocéntricos y el exceso de confianza en el Sistema 1 para la administración de justicia se explican por sí solos. La justicia requiere combatirlos.

\subsection{Aversión a los extremos}

Por último, trataremos sobre el sesgo de aversión a los extremos. A las personas les incomodan las situaciones extrapoladas, prefieren los términos medios. En el mundo arbitral, de hecho, es común escuchar la crítica de que los árbitros prefieren emitir laudos "salomónicos", dando parcialmente la razón a ambas partes. En una encuesta realizada por la Universidad Queen Mary se observó que una de las críticas más comunes al sistema arbitral es que los tribunales tienden a emitir laudos salomónicos (splitting

27. R. WAites y J. LaWrence, "Psychological Dynamics in International Arbitration Advocacy" en D. BisHOP y E. KeHOE (Eds.), The Art of Advocacy in International Arbitration, p. 114. Citado por E. SusSMAN, Ibídem, p. 502. 
the baby) de manera innecesaria, siendo "reacios a fallar fuertemente en favor de una parte" 28 .

A criterio del profesor AlBERT JAN VAN DEN BERG, la creencia de que los árbitros adoptan decisiones salomónicas se debería a varias razones, entre ellas, al método de selección de los árbitros. Cuando cada parte nombra a "su" árbitro, podría generarse la idea errónea de que el árbitro defiende los intereses de la parte que lo designó. Esto llevaría al presidente del tribunal a encontrar un punto medio entre las posiciones de sus coárbitros. Otra razón sería el hecho de que las partes suelen inflar las cuantías de sus reclamos. Si el árbitro no concede el ciento por ciento del monto reclamado, las partes podrían pensar que el árbitro tomó una decisión equilibrada ${ }^{29}$.

Si bien la aversión a los extremos es un patrón psicológico del que podríamos padecer ${ }^{30}$, las buenas prácticas arbitrales parecen haberlo mitigado en gran medida. Estudios empíricos recientes demostrarían que (al menos en arbitraje internacional), los árbitros no suelen ser salomónicos. Por ejemplo, KEER y NAIMARK analizaron ciento once laudos emitidos entre los años 1995 y 2000 bajo las reglas de la American Arbitration Association. El estudio demuestra que en el $66 \%$ de los casos los árbitros otorgaron todo o nada de la indemnización reclamada, y solo en el 34\% restante se otorgaron cuantías que fluctuaron entre el 10\% y el $90 \%$ de los montos reclamados. En consecuencia, estos autores concluyeron que "al parecer, existe poco soporte fáctico sobre la idea de que los árbitros parten irrazonablemente los montos de los laudos" ${ }^{\prime 31}$.

28. Queen Mary University of London, School of International Arbitration (SIA) y White \& Case, "2012 International Arbitration Survey: Current and Preferred Practices in the Arbitral Process”, p. 38, «https://goo.gl/i3qZqo». Nótese, sin embargo, que el estudio revela que solo en el $17 \%$ de los casos los árbitros dictan laudos salomónicos.

29. A. Jan Van Den Berg, Decisiones salomónicas: Realidades, mitos y ética (Ponencia de 5/12/2013), Centro de Arbitraje de la Industria de la Construcción, diciembre de 2013, «https://goo.g1/zAiTpW».

30. Existe una serie de estudios sobre este tema. Véase por ejemplo, A. CHERNEV, "Extremeness Aversion and Attribute-Balance Effects in Choice", Journal of Consumer Research, 2004, «https://goo.gl/ dQtZCP»; y, I. SiMONSON y A. TVERSKY, N. 18.

31. Traducción libre. S. Keer y R. NAIMARK, “Arbitrators Do Not 'Split the Baby'-Empirical Evidence from International Business Arbitrations”, Journal of International Arbitration, 2001, pp. 577-578. 
A una conclusión similar arribó MENTSCHIKOFF, quien verificó que en el 50\% de un grupo de laudos analizados los árbitros concedieron todo o nada de la indemnización demandada ${ }^{32}$.

Para LuCy ReED, la razón para que los árbitros no emitan laudos salomónicos es su disciplina en la aplicación de la ley y en la motivación de los laudos ${ }^{33}$. En la siguiente sección de este trabajo propondremos mecanismos para sistematizar tal disciplina.

\section{4. ¿Cómo MejORAR LAS DECISIONES ARBITRALES?}

Estar conscientes de nuestros sesgos, prejuicios y defectos es un buen primer paso. Sin embargo, nuestra consciencia no es suficiente para evitarlos. Estudios psicológicos demuestran, de hecho, que las personas suelen creer que su pensamiento no se encuentra afectado por tales vicios (aunque estén conscientes de su existencia), generándose un nuevo vicio denominado el "punto ciego del sesgo" o "bias blind spot"

Las personas que se encuentran embestidas de la delicada tarea de administrar justicia (o resolver controversias) tienen el deber de hacer sus mejores esfuerzos por proferir fallos justos, apegados a derecho y a los hechos del caso. Si bien la psicología experimental y neurociencia aún no se encuentran en un estado de desarrollo tal que permita conocer con certeza cómo neutralizar nuestros sesgos, existen varias técnicas que podrían servir de conjuros. A continuación resumimos algunas de ellas ${ }^{35}$.

32. S. MentschikofF, “Commercial Arbitration”, Columbia Law Review 846, No. 61, 1961, «https://goo.gl/ iv1RJZ».

33. L. REED, N. 25, p. 9.

34. Sobre este tema véase, por ejemplo, a E. Pronin, D. Linn y L. Ross, "The Bias Blind Spot: Perceptions of Bias in Self Versus Others", Personality and Social Psychology Bulletin, Vol. 28 (3), 2002, p. 369.

35. Para realizar este listado de técnicas se ha tomado en consideración los consejos de los autores citados en este artículo. Otros son de autoría propia. 


\subsection{Controlar el tiempo}

Una de las maneras más efectivas de combatir nuestros sesgos y, por lo tanto, la probabilidad de fallos equívocos o injustos, es inducir la activación del Sistema 2. Para ello, es indispensable que el árbitro tenga suficiente tiempo para pensar. Si el Sistema 2 del árbitro se encuentra permanentemente sobrecargado, distraído, con poco tiempo, la probabilidad de que el Sistema 1 defina la controversia se incrementa.

Si lo que propone la psicología experimental y la neurociencia contemporáneas sobre el tiempo que necesitan los juzgadores para fallar adecuadamente llegare a comprobarse, el esquema de juzgamiento concebido por el Código Orgánico General de Procesos (COGEP) sería preocupante. En general, el COGEP privilegiaría la rapidez de la decisión por sobre el análisis, pues manda que los jueces resuelvan la controversia en la misma audiencia de juicio ${ }^{36}$. Ya tomada la decisión, el juez la redacta posteriormente ${ }^{37}$. Esta metodología parecería ajustarse a la escuela realista a la que nos referimos anteriormente (solo que sin el trasfondo filosófico que la sustenta) $)^{38}$, bajo la cual los jueces fallarían con base a sus acorazonadas (porque no tendrían tiempo para emprender un ciclo analítico completo, por más experimentados que los jueces sean), siendo la fase de redacción de la sentencia una mera formalidad destinada a dar forma a sus decisiones intuitivas. Afortunadamente, el sistema intuitivo que propone el COGEP no debería afectar al arbitraje, pues el proceso de juzgamiento arbitral se encuentra delineado por la Ley de Arbitraje y Mediación (LAM) y no por el COGEP, al menos en lo que a alegaciones y tiempos de resolución se refiere ${ }^{39}$.

36. Por ejemplo, el Art. 79 del COGEP dispone: "se resolverá de manera motivada en la misma audiencia. Las personas serán notificadas con el solo pronunciamiento oral de la decisión".

37. Por ejemplo, el Art. 93 del COGEP señala: "Pronunciamiento judicial oral. Al finalizar la audiencia la o al juzgador pronunciará su decisión en forma oral. Excepcionalmente y cuando la complejidad del caso lo amerite podrá suspender la audiencia por el término de hasta diez días para emitir su decisión oral. Al ordenar la suspensión determinará el día y la hora de reinstalación de la audiencia. La resolución escrita motivada se notificará en el término de hasta diez días".

38. No existen evidencias de que el legislador haya privilegiado la velocidad de las decisiones por razones filosóficas o de ciencia jurídica. Tampoco existen evidencias de que el legislador haya evaluado la eventual afectación que este sistema podría tener en la calidad de la administración de justicia.

39. Según el Art. 37 de la LAM, el COGEP es aplicable al arbitraje únicamente (i) de manera supletoria y (ii) siempre que el proceso sea en derecho. Aplicando concurrentemente el Art. 38 de la LAM, entonces, 
A continuación proponemos algunas técnicas para que los árbitros controlen el tiempo y propicien un proceso analítico:

- No permitir que el tiempo que el tribunal tiene para fallar sea consumido por las partes. Esto implica, entre otras cosas, controlar incidentes y el exceso de litigiosidad, evitar pruebas y audiencias inoficiosas, solicitar a las partes que sus argumentaciones no sean demasiado extensas, coordinar tempranamente con los coárbitros sus agendas y obligar a que los peritos realicen prontamente sus informes. Idealmente, las pruebas y audiencias finales (ej. audiencia en estrados) deberían terminar varios meses antes de que el tribunal emita su laudo.

- El tribunal podría preparar un calendario completo del caso, hasta su conclusión, en el cual se defina el tiempo que necesitará para analizar los argumentos y las pruebas de las partes, redactar el laudo, consultarlo y revisarlo. A este efecto, el tribunal debería analizar tempranamente la complejidad y extensión del caso.

- Evitar tomar demasiados casos. El número máximo de casos que un árbitro debería tomar es aquel que le permita dedicar suficiente tiempo a todos ellos.

- Buscar lugares donde, fuera del ruido y la vertiginosidad del trabajo, el árbitro pueda concentrarse y redactar su decisión, sin distracciones.

- Dejar "madurar" al laudo; esto es, redactarlo y separarse de él por un período de tiempo, para luego volverlo a revisar con mente fresca.

\subsection{Razonar con el Sistema 2}

Como hemos comentado insistentemente, los sesgos más recurrentes del juzgador se presentarían en la fase cognitiva del juz-

el COGEP sería aplicable supletoriamente sólo si, en un arbitraje en derecho, (i) la LAM, (ii) los reglamentos de los Centros; y (iii) las reglas pactadas entre las partes, no regulan una situación procesal específica. La audiencia de sustanciación, los alegatos en estrados, el término para proferir el laudo, entre otras normas procesales que protegen al ciclo cognitivo del tribunal, se encuentran regulados por la LAM, lo que hace innecesario (e improcedente) acudir a una norma supletoria. 
gamiento. A continuación enumeramos algunas propuestas para combatir los sesgos del razonamiento apoyándonos en el Sistema 2:

- Identificar tempranamente los puntos sensibles del fallo y los posibles errores que se podrían cometer.

- Identificar las distintas interpretaciones que se podría dar al caso, de manera que no se subestime otras explicaciones a los hechos, y se verifique si no existe una aplicación distinta o más apropiada de la ley.

- Al momento de redactar el laudo, el árbitro podría ponerse en la posición de las dos partes, asumiendo hipotéticamente que a ambas partes les asistiría la razón, de manera que se verifique la solidez de ambas teorías del caso y no solo de la parte a favor de la cual fallará.

- Identificar las pruebas que no son confiables y que podrían llevar al árbitro a un fallo erróneo.

- Consultar y repasar con los coárbitros (si el tribunal es colegiado) todos los aspectos de hecho y derecho del caso, evitando que el caso sea manejado solo por una persona.

- Hacer un listado de los hechos que benefician a cada una de las partes, a fin de no pasarlos desapercibidos.

- Hacer un listado de las pretensiones y excepciones de cada parte, verificando que todas hayan sido abordadas con la misma profundidad.

- Evitar confiar solamente en la memoria; referirse a las fuentes y pruebas originarias de ambas partes, de manera que se evite la malinterpretación de los hechos.

- Para lo anterior, se sugiere elaborar anexos con todas las pruebas del proceso, de manera que no se pase a ninguna por alto.

- Pensar qué prueba podría haber definido el caso en sentido contrario y asegurarse de que tal prueba no exista.

- Pensar qué argumentos de hecho o derecho no estuvieron presentes para fallar a favor de la otra parte.

- Pensar qué argumentos utilizarían las partes para intentar anular el laudo, a fin de evitar errores de debido proceso. 
- Identificar si existen situaciones o razonamientos en los que los sesgos de anclaje, retrospectiva, ego y aversión a los extremos, entre otros, podrían estar presentes. Por ejemplo, si se debe valorar la posibilidad de predecir un hecho, se debe tener presente el sesgo de retrospectiva y combatirlo activamente. Si se deben definir montos indemnizatorios, se sugiere evitar conscientemente las anclas y la aversión a los extremos (especialmente las cuantías propuestas por las partes) y realizar una valoración propia. Ante los sesgos de ego, confiar más en los coárbitros y menos en el buen juicio personal es un buen comienzo, manteniendo la mente siempre abierta para no prejuzgar la controversia.

\subsection{Redactar el laudo minuciosamente}

Obligarse a motivar suficientemente la decisión, no solo para cumplir los estándares legales, sino para que el tribunal y las partes comprendan adecuadamente el razonamiento que subyace al fallo, permite que el Sistema 2 entre en funcionamiento. La redacción motivada del laudo propicia un proceso cuidadoso, lógico y deductivo. Existen algunas técnicas:

- Elaborar tempranamente un bosquejo del laudo en el que se incluyan todas las cuestiones jurídicas relevantes. Esto obligará al tribunal a analizar todos los puntos por igual, evitando saltar a los que más le gusten, le llamen más la atención o conozca más. Esto también ayudará a evitar laudos con incongruencias procesales (e.i. infra o extra petita).

- Tomar nota permanentemente de las ideas que vienen a la mente al árbitro, especialmente en las audiencias y diligencias probatorias. La lluvia de ideas constante es parte vital del ciclo cognitivo. Esto permitirá que el proceso de redacción sea continuo, evitando empezar a escribir la decisión recién cuando el caso está por concluir.

- Los laudos deberían tener una sección detallada y cronológica de los hechos, a los cuales se les aplicará el Derecho. Los hechos deberían encontrarse sustentados en las pruebas pro- 
ducidas en el proceso y no solo en las argumentaciones de las partes. Los hechos no probados no deberían ser considerados.

- Escribir con claridad, evitando el lenguaje difícil. Cualquier persona debería ser capaz de comprender la motivación del laudo. Esforzarnos por escribir de manera simple activa el Sistema 2, pues plasmar un razonamiento jurídico complejo en palabras sencillas requiere método, concentración y capacidad de síntesis.

\section{Conclusiones}

Este artículo propone un debate sobre la potencial aplicación de los recientes estudios psicológicos y neurocientíficos a la práctica del arbitraje en Ecuador. Los indicios de que los árbitros no siempre aplican el derecho de manera mecánica, fría y lógica, sumados a las señales de que el pensamiento del árbitro puede verse aquejado por varios sesgos, nos invita a reflexionar sobre las técnicas que se podrían adoptar para mejorar las decisiones arbitrales.

Si bien la administración de justicia pública y la resolución de conflictos privada nunca serán sistemas perfectos, la trascendencia del rol del juzgador para la sociedad compele a conocer mejor cómo funciona nuestra mente, a fin de precautelar la calidad de la justicia.

En suma, este artículo propone que la mayoría de sesgos cognitivos de los árbitros podrían verse relativamente conjurados activando el Sistema 2, para lo cual los árbitros necesitan gozar de suficiente tiempo para inducir un ciclo analítico apropiado.

Se espera que un día las personas empiecen a preferir al arbitraje por sobre la justicia ordinaria, no solo por sus ventajas conocidas del primero (neutralidad, rapidez, especialidad, inapelabilidad de laudos, etc.), sino porque se aspiraría a que los 
árbitros dediquen más tiempo que un juez a pensar y resolver sus casos. 